\title{
A Remark on Atomic Decompositions of Martingale Hardy's Spaces
}

\author{
Maciej Paluszynski ${ }^{1}$ (D) Jacek Zienkiewicz ${ }^{1}$
}

Received: 12 March 2020 / Published online: 27 June 2020

(c) The Author(s) 2020

\section{Abstract}

We prove theorems and exhibit a counterexample concerning an atomic decomposition of martingale $\mathbb{H}^{1}$ with atoms satisfying simultaneous cancellation condition (3).

Keywords Hardy space · Atomic decomposition · Dyadic martingale

Mathematics Subject Classification 42B25 $\cdot 11 \mathrm{P} 05$

\section{Introduction}

Let $f \in L^{1}(\mathbb{R})$. We say that $f \in \mathbb{H}^{1}$ (dyadic martingale Hardy space) if

$$
M f(x)=\sup _{I: x \in I} \frac{1}{|I|}\left|\int_{I} f\right| \in L^{1}, \quad\|f\|_{\mathbb{H}^{1}}=\|M f\|_{L^{1}}
$$

where the supremum is taken over all dyadic intervals $I$ containing $x$. The basic property of Hardy spaces is the so called atomic decomposition. We say that a function $a_{I}$ is a (dyadic) atom, or an $\mathbb{H}^{1}$ - atom, associated with a dyadic interval $I$ if

- $\operatorname{supp} a_{I} \subset I$,

- $\left\|a_{I}\right\|_{L^{\infty}} \leq \frac{1}{|I|}$,

- $\int a_{I}=0$.

Dedicated to Guido Weiss on the occasion of his 90th Birthday.

$\bowtie \quad$ Maciej Paluszynski

mpal@math.uni.wroc.pl

Jacek Zienkiewicz

zenek@math.uni.wroc.pl

1 Instytut Matematyczny, Uniwersytet Wrocławski, pl. Grunwaldzki 2/4, 50-384 Wrocław, Poland 
Any function $f \in \mathbb{H}^{1}$ admits a decomposition

$$
f=\sum_{I} \lambda_{I} a_{I}, \quad \text { in } \mathbb{H}^{1},
$$

where $a_{I}$ are atoms and

$$
\sum_{I}\left|\lambda_{I}\right| \leq C\|f\|_{\mathbb{H}^{1}}
$$

The martingale Hardy spaces were first introduced in [3], with atomic decomposition implicitly appearing in [4], and the explicit proof appearing in [1]. More on the subject of atomic decompositions in the martingale setting can be found in [8]. Classical introduction to Hardy spaces can be found in [7]. Atomic decompositions in the classical case were developed in [2] and [5]. In this note we address the following question: Suppose we are given two weights $w_{1}, w_{2}$ on $\mathbb{R}$ and $f \in \mathbb{H}^{1}$ satisfying

$$
1 \leq w_{i} \leq C, \quad w_{i} \cdot f \in \mathbb{H}^{1}, i=1,2 .
$$

Can one obtain a decomposition (2) with atoms $a_{I}$ satisfying simultaneously

$$
\int a_{I} \cdot w_{1}=\int a_{I} \cdot w_{2}=0 ?
$$

One of the main results of this note is a negative answer to this natural question.

We also give a maximal function characterization of those $f \in \mathbb{H}^{1}$ which admit decomposition (2) with atoms $a_{I}$ satisfying (3).

\section{The Results}

Given a weight function $w$ we call $w \mathbb{H}^{1}$ the space $\left\{f: w \cdot f \in \mathbb{H}^{1}\right\}$ with norm $\|f\|_{w \mathbb{H}^{1}}=\|w \cdot f\|_{\mathbb{H}^{1}}$. A function $a$ is called a $w \mathbb{H}^{1}$ atom if $w \cdot a$ is an atom. Since the weights $w$ we consider are bounded and bounded away from 0 , the only difference between an atom and a $w \mathbb{H}^{1}$ atom is in the cancellation condition.

Theorem 1 There exist weights $1 \equiv w_{1} \leq w_{2} \leq c$ and a function $f$ with $w_{1} f \in \mathbb{H}^{1}$ and $w_{2} f \in \mathbb{H}^{1}$, which does not have a joint atomic decomposition, that is it does not have a decomposition

$$
f=\sum \lambda_{Q} b_{Q}, \quad \sum\left|\lambda_{Q}\right| \leq C \max \left\{\|f\|_{w_{1} \mathbb{H}^{1}},\|f\|_{w_{2} \mathbb{H}^{1}}\right\}
$$

with $b_{Q}$ being both $w_{1} \mathbb{H}^{1}$ and $w_{2} \mathbb{H}^{1}$ atoms.

Remark Theorem is stated for $w_{1} \equiv 1$. It is clear, that the same construction can be applied to the case of arbitrary $w_{1} \sim 1$. That is for any weight $w_{1} \sim 1$ there exists a $w_{2}$ satisfying $w_{1} \leq w_{2} \leq C$, such that the theorem holds. 
Proof Consider the interval $[0,1]$ and arbitrary $n \in \mathbb{N}$. Let $I_{k}$ be consecutive, adjacent intervals of length $2^{-k}$

$$
I_{k}=\left[\frac{2^{k}-2}{2^{k}}, \frac{2^{k}-1}{2^{k}}\right], \quad k=1,2, \ldots, n .
$$

Each of these intervals is an element of the standard dyadic grid. The left half of $I_{k}$ is denoted by $I_{k}^{+}$and the right half by $I_{k}^{-}$:

$$
I_{k}^{+}=\left[\frac{2^{k}-2}{2^{k}}, \frac{2^{k}-3 / 2}{2^{k}}\right], \quad I_{k}^{-}=\left[\frac{2^{k}-3 / 2}{2^{k}}, \frac{2^{k}-1}{2^{k}}\right] .
$$

We define:

$$
a_{I_{k}}(x)= \begin{cases}2^{k}\left(1+\frac{1}{n}\right)^{k-1} & : x \in I_{k}^{+}, \\ -2^{k}\left(1+\frac{1}{n}\right)^{k-1} & : x \in I_{k}^{-}, \\ 0 & : x \notin I_{k} .\end{cases}
$$

The functions $a_{I_{k}}$ satisfy:

- $\operatorname{supp} a_{I_{k}} \subset I_{k}$,

- $\int a_{I_{k}}=0$,

- $\left\|a_{I_{k}}\right\|_{L^{\infty}}=2^{k}\left(1+\frac{1}{n}\right)^{k-1}=\frac{\left(1+\frac{1}{n}\right)^{k-1}}{\left|I_{k}\right|} \leq \frac{e}{\left|I_{k}\right|}$,

that is they are $e$ - multiples of $\mathbb{H}^{1}$ atoms. We let

$$
f_{0}=\sum_{k=1}^{n} a_{I_{k}}
$$

and thus

$$
\left\|f_{0}\right\|_{\mathbb{H}^{1}} \leq \sum_{k=1}^{n}\left\|a_{I_{k}}\right\|_{\mathbb{H}^{1}} \leq C n
$$

Let us define the weight $w_{2}$

$$
w_{2}(x)= \begin{cases}\left(1+\frac{1}{n}\right) & : x \in I_{k}^{-}, \quad k=1,2, \ldots, \\ 1 & : x \notin \bigcup I_{k}^{-} .\end{cases}
$$

We obtain the decomposition

$$
w_{2} \cdot f_{0}=2 \mathbb{1}_{I_{1}^{+}}-2^{n} \cdot\left(1+\frac{1}{n}\right)^{n} \mathbb{1}_{I_{n}^{-}}+\sum_{k=1}^{n-1} b_{J_{k}},
$$


where

$$
J_{k}=I_{k}^{-} \cup I_{k+1}^{+},
$$

and

$$
b_{J_{k}}(x)= \begin{cases}-2^{k}\left(1+\frac{1}{n}\right)^{k} & : x \in I_{k}^{-} \\ 2^{k+1}\left(1+\frac{1}{n}\right)^{k} & : x \in I_{k+1}^{+} \\ 0 & : x \notin J_{k}\end{cases}
$$

Observe, that $b_{J_{k}}$ are $3 e / 2$ multiples of $\mathbb{H}^{1}$ atoms.

- $\operatorname{supp} b_{J_{k}}=J_{k}$,

- $\int b_{J_{k}}=-\frac{1}{2}\left(1+\frac{1}{n}\right)^{k}+\frac{1}{2}\left(1+\frac{1}{n}\right)^{k}=0$,

- $\left\|b_{J_{k}}\right\|_{L^{\infty}}=2^{k+1}\left(1+\frac{1}{n}\right)^{k}=\frac{3\left(1+\frac{1}{n}\right)^{k}}{2\left|J_{k}\right|} \leq \frac{3 e}{2\left|J_{k}\right|}$.

Note that

$$
J_{k}=I_{k}^{-} \cup I_{k+1}^{+}=\left[1-\frac{3}{2^{k+1}}, 1-\frac{3}{2^{k+2}}\right] \subset\left[1-\frac{1}{2^{k-1}}, 1\right]=\tilde{J}_{k},
$$

where $\tilde{J}_{k}$ is an element of the standard dyadic grid, with length comparable to that of $J_{k}$

$$
\left|J_{k}\right|=\frac{3}{2^{k+2}}, \quad\left|\tilde{J}_{k}\right|=\frac{1}{2^{k-1}} .
$$

Thus

$$
\left\|\sum_{k=1}^{n-1} b_{J_{k}}\right\|_{\mathbb{H}^{1}} \leq \sum_{k=1}^{n-1}\left\|b_{J_{k}}\right\|_{\mathbb{H}^{1}} \leq C n
$$

To account for the remaining parts of $w_{2} \cdot f_{0}$ we extend $f_{0}$ to $[1,2]$ and modify $w_{2}$ there. Let

$$
\alpha=\int f_{0} w_{2}=\frac{1}{2}-\frac{1}{2}\left(1+\frac{1}{n}\right)^{n}, \quad-1 \leq \alpha \leq-\frac{1}{2} .
$$

For $x \in[1,2]$ we let

$$
w_{2}(x)= \begin{cases}1 & : x \in\left[1, \frac{3}{2}\right] \\ \left(1+\frac{1}{n}\right) & : x \in\left(\frac{3}{2}, 2\right]\end{cases}
$$


and

$$
f(x)= \begin{cases}f_{0}(x) & : x \in[0,1] \\ 2 \alpha n & : x \in\left[1, \frac{3}{2}\right] \\ -2 \alpha n & : x \in\left(\frac{3}{2}, 2\right] \\ 0 & : x \notin[0,2]\end{cases}
$$

Then

$$
\|f\|_{\mathbb{H}^{1}} \leq\left\|f_{0}\right\|_{\mathbb{H}^{1}}+2|\alpha| n \leq C n .
$$

Moreover $w_{2} \cdot f$ decomposes as

$$
w_{2} \cdot f=\sum_{k=1}^{n-1} b_{J_{k}}+A
$$

where

$$
A=2 \mathbb{1}_{I_{1}^{+}}-2^{n}\left(1+\frac{1}{n}\right)^{n} \mathbb{1}_{I_{n}^{-}}+2 \alpha n \mathbb{1}_{[1,3 / 2]}-2 \alpha n\left(1+\frac{1}{n}\right) \mathbb{1}_{(3 / 2,2]} .
$$

It is a straightforward argument to show that $\|A\|_{\mathbb{H}^{1}} \leq C n$. Similar straightforward computation shows the same estimate holds for the classical, non-martingale Hardy's space. We will comment on this later. Thus

$$
\left\|w_{2} \cdot f\right\|_{\mathbb{H}^{1}} \leq\left\|\sum_{k=1}^{n-1} b_{J_{k}}\right\|_{\mathbb{H}^{1}}+\|A\|_{\mathbb{H}^{1}} \leq C n
$$

We have just shown

$$
\|f\|_{w_{1} \mathbb{H}^{1}} \leq C n, \quad\|f\|_{w_{2} \mathbb{H}^{1}} \leq C n
$$

Consider the function

$$
\begin{aligned}
g(x) & =n\left(w_{1}(x)-w_{2}(x)\right) \cdot f(x) \\
& = \begin{cases}2^{k}\left(1+\frac{1}{n}\right)^{k-1} & : x \in I_{k}^{-}, \quad k=1,2, \ldots, n \\
2 \alpha n & : x \in\left(\frac{3}{2}, 2\right], \\
0 & : \text { otherwise. }\end{cases}
\end{aligned}
$$

We will show, that

$$
\|g\|_{\mathbb{H}^{1}}=\|M g\|_{L^{1}} \geq C n^{2} .
$$


Let us consider $x \in I_{k}^{-}$. Immediate dyadic parents of $I_{k}^{-}$are

$$
I_{k}, \quad\left[1-2^{-i}, 1\right], i=0,1, \ldots, k-1
$$

We compute the average of $g$ over $I=\left[1-2^{-(k-1)}, 1\right]$.

$$
\begin{aligned}
\frac{1}{|I|} \int_{I} g(y) d y & =2^{k-1} \sum_{i=k}^{n} \int_{I_{i}^{-}} 2^{i}\left(1+\frac{1}{n}\right)^{i-1} d y \\
& =2^{k-1} \sum_{i=k}^{n} 2^{-(i+1)} 2^{i}\left(1+\frac{1}{n}\right)^{i-1} \\
& =2^{k-2} \sum_{i=k-1}^{n-1}\left(1+\frac{1}{n}\right)^{i} \\
& =2^{k-2} n\left(\left(1+\frac{1}{n}\right)^{n}-\left(1+\frac{1}{n}\right)^{k-1}\right) .
\end{aligned}
$$

Thus

$$
M g(x) \geq 2^{k-2} n\left(\left(1+\frac{1}{n}\right)^{n}-\left(1+\frac{1}{n}\right)^{k-1}\right), \quad x \in I_{k}^{-} .
$$

Integrating $M g$ we obtain

$$
\|M g\|_{L^{1}} \geq \sum_{k=1}^{n}\left|I_{k}^{-}\right| 2^{k-2} n\left(\left(1+\frac{1}{n}\right)^{n}-\left(1+\frac{1}{n}\right)^{k-1}\right)=\frac{n^{2}}{8}
$$

In fact, we can show a stronger estimate, namely $\|g\|_{\mathbb{H}^{1}} \geq C n^{2}$, where the norm is in the classical Hardy's space. We will comment on that in a remark below. To see this stronger estimate, let us fix a test function

$$
\mathbb{1}_{[-1,1]} \leq \Phi \leq \mathbb{1}_{[-3 / 2,3 / 2]}
$$

Then

$$
\begin{aligned}
\Phi_{t} * g(x) & =\left(\Phi_{t} * \sum_{k=1}^{n} 2^{k}\left(1+\frac{1}{n}\right)^{k-1} \mathbb{1}_{I_{k}^{-}}\right)(x)+\left(\Phi_{t} *\left(2 \alpha n \mathbb{1}_{(3 / 2,2]}\right)\right)(x) \\
& =\left(\Phi_{t} * F\right)(x)+\left(\Phi_{t} * G\right)(x), \\
& =\frac{1}{t} \int_{x-\frac{3}{2} t}^{x+\frac{3}{2} t} \Phi\left(\frac{x-y}{t}\right) F(y) d y+\frac{1}{t} \int_{x-\frac{3}{2} t}^{x+\frac{3}{2} t} \Phi\left(\frac{x-y}{t}\right) G(y) d y
\end{aligned}
$$


where

$$
\operatorname{supp} F=\bigcup_{k=n / 2}^{n} I_{k}^{-} \subset\left[1-3 \cdot 2^{-n / 2-1}, 1\right], \quad \operatorname{supp} G=(3 / 2,2]
$$

Now, take $x \in\left[0,1-3 \cdot 2^{-n / 2}\right](n \geq 4)$, and $t=1-x$. Then the second integral vanishes due to disjoint supports. Thus

$$
\Phi_{t} * g(x)=\frac{1}{t} \int_{x-\frac{3}{2} t}^{x+\frac{3}{2} t} \Phi\left(\frac{x-y}{t}\right) F(y) d y \geq \frac{1}{t} \int_{x-t}^{x+t} F(y) d y
$$

Since $t=1-x$, we have $x+t=1$ and $x-t=2 x-1 \leq 1-3 \cdot 2^{-n / 2-1}$, so the integration interval covers the entire support of $F$. Thus, in the chosen range of $x$

$$
\begin{aligned}
|M g(x)| & \geq\left|\Phi_{1-x} * g(x)\right| \\
& \geq \frac{1}{1-x} \int_{2 x-1}^{1} F(y) d y \\
& =\frac{1}{1-x} \sum_{k=n / 2}^{n}\left(1+\frac{1}{n}\right)^{k-1} \cdot \frac{1}{2} \\
& \geq \frac{1}{1-x} \cdot \frac{n}{4} .
\end{aligned}
$$

Consequently,

$$
\begin{aligned}
\|M g\|_{L^{1}} & \geq \int_{0}^{1-3 \cdot 2^{-n / 2}}|M g(x)| d x \\
& \geq \frac{n}{4} \int_{0}^{1-3 \cdot 2^{-n / 2}} \frac{d x}{1-x} \\
& =\frac{n}{4} \cdot\left(\frac{n}{2} \log 2-\log 3\right) \\
& \geq C n^{2}
\end{aligned}
$$

We continue with the proof of the theorem. Suppose $f$ does have a decomposition

$$
f=\sum_{Q} \lambda_{Q} b_{Q}
$$

where $b_{Q}$ are both $w_{1} \mathbb{H}^{1}$ and $w_{2} \mathbb{H}^{1}$ atoms, as in the statement of the theorem. Then, by estimates above

$$
\sum_{Q}\left|\lambda_{Q}\right| \leq C n
$$


This would imply

$$
\begin{aligned}
\|g\|_{\mathbb{H}^{1}} & =\left\|n\left(w_{1}-w_{2}\right) \sum_{Q} \lambda_{Q} \cdot b_{Q}\right\|_{\mathbb{H}^{1}} \\
& \leq \sum_{Q}\left|\lambda_{Q}\right| \cdot\left\|n\left(w_{1}-w_{2}\right) b_{Q}\right\|_{\mathbb{H}^{1}} \\
& \leq C n,
\end{aligned}
$$

since each $n\left(w_{1}-w_{2}\right) b_{Q}$ is an $\mathbb{H}^{1}$ atom:

- $\operatorname{supp} n\left(w_{1}-w_{2}\right) b_{Q} \subset Q$,

- $\int n\left(w_{1}-w_{2}\right) b_{Q}=0$,

- $\left\|n\left(w_{1}-w_{2}\right) b_{Q}\right\|_{L^{\infty}} \leq\left\|b_{Q}\right\|_{L^{\infty}} \leq \frac{1}{|Q|}$.

Thus, since the constants are independent of $n$, we have obtained a contradiction

$$
n^{2} \lesssim\|g\|_{\mathbb{H}^{1}} \lesssim n
$$

We call just constructed function $f_{n}$, and the weight $w^{n}$. Both are localized on $[0,2]$. It is now routine to appropriately $\mathbb{H}^{1}$-scale and shift thus constructed $f_{n}$ 's, together with $w^{n}$ 's (both operations necessarily dyadic), so they are all localized within $[0,1]$, with disjoint supports. The sum of $n^{-\frac{3}{2}} f_{n}$ 's over a dyadic $n$ 's, together with weight $w$, being the sum of $w^{n}$ 's is the required example for which the condition (4) cannot hold. This completes the proof.

Remark Observe, that the above theorem is also valid in the case of classical Hardy's space.

We point to another possible construction of the weight $w$ from Theorem 1, very much in the spirit of tweaks known from the theory of Cauchy Integral. Let $I \subset[0,1]$ be dyadic. We let $w^{n}$ be given by (5) below (modified weight from the above theorem), and denote by $w_{I}^{n}$ this weight re-scaled and translated to $I$. Suppose $\left\{n_{k}\right\}$ is a sequence of naturals increasing to infinity sufficiently fast. We construct a sequence of weights $\omega^{k}$.

(i) We put $\omega^{1}=w_{[0,1]}^{n_{1}}$.

(ii) Assume $\omega^{1}, \ldots, \omega^{k}$ have already been constructed. Let $I_{k, j}, j=1, \ldots, l_{k}$ be the maximal dyadic intervals on which $\omega^{k}=c_{k, j}$ is constant. Then, for $x \in I_{k, j}$ we put $\omega^{k+1}(x)=c_{k, j} w_{I_{k, j}}^{n_{k+1}}(x)$. Observe that by construction $\bigcup_{j} I_{k, j}=[0,1]$ (iii) We put $w(x)=\lim _{k \rightarrow \infty} \omega^{k}(x)$.

The weight obviously satisfies requirements of the Theorem 1 (together with the function $f$, which consists of parts constructed in the proof, but summed differently). We also point out (we leave the proof to the reader), that it satisfies the condition (6) below. 
We will now prove a maximal function characterization of those functions on $[0,1]$ that do admit atomic decomposition with atoms satisfying double cancellation condition. From now on we fix $n$ and the weights $w_{1} \equiv 1$ and $w_{2}=w$ on $[0,1]$ constructed in the proof of Theorem 1 . Let us recall

$$
w(x)= \begin{cases}\left(1+\frac{1}{n}\right) & : x \in I_{k}^{-}, \quad k=1,2, \ldots, \\ 1 & : x \notin \bigcup I_{k}^{-},\end{cases}
$$

(we do no restrict $k$ to be $\leq n$, thus $\operatorname{supp} w=[0,1]$ ). We will state a quantitative version of our result for these weights. The argument clearly extends to any pair $w_{1}, w_{2}$ satisfying condition (6) below, with $w=w_{2} w_{1}^{-1}$. Typical examples of such weights are those defined by lacunary Fourier series or positive polynomials. See Corollary following Theorem 2 .

Let us recall that we are working in the setting of the standard dyadic martingale on $\mathbb{R}$. Our aim is to define a maximal function which would characterize an atomic space with atoms simultaneously orthogonal to both 1 and $w$. We put

$$
\Delta_{I}(x)=\mathbb{1}_{I}(x)\left(\beta_{I}-w(x)\right)
$$

with the constant $\beta_{I}$ chosen so that $\int \Delta_{I}=0$. Obviously,

$$
\frac{\mathbb{1}_{I}(x)}{|I|^{1 / 2}}, \quad \frac{\Delta_{I}(x)}{\left\|\Delta_{I}\right\|_{L^{2}(I)}}
$$

are orthonormal functions in $L^{2}(I)$, obtained by Gramm-Schmidt orthogonalization of weights $\mathbb{1}_{I}$ and $\mathbb{1}_{I} \cdot w$ on $I$. We define the following maximal operator

$$
\mathcal{M} f(x)=\sup _{\substack{I \subset[0,1]-\text { dyad. } \\ \alpha \in \mathbb{R}}} \frac{\mathbb{1}_{I}(x)}{|I| \cdot\|\alpha-w\|_{L^{\infty}(I)}}\left|\int_{I} f \cdot(\alpha-w)\right|
$$

It is immediate that $\mathcal{M} f \leq C M_{H L} f$, where $M_{H L} f$ is the standard Hardy-Littlewood maximal function on $[0,1]$. We will use the following

Lemma Let $I \subset[0,1]$. Then, for some constant $C$ independent on $n$, we have

$$
\left|\frac{\Delta_{I}(x)}{\left\|\Delta_{I}\right\|_{L^{2}}^{2}} \int_{I} f \cdot \Delta_{I}\right| \leq C \mathcal{M} f(x)
$$

Proof The lemma follows immediately from the following condition satisfied by the weight $\alpha-w$ : there is the constant $C$ independent of $\alpha$ and $n$ such that for any dyadic interval $I$ we have

$$
|I| \cdot\|\alpha-w\|_{L^{\infty}(I)}^{2} \leq C\|\alpha-w\|_{L^{2}}^{2}
$$

To see this, suppose $I \subset[0,1]$ is a dyadic interval. Since $\bigcup_{k \geq 1} I_{k}=[0,1)$ one of the following cases has to hold. 
(i) There exists a $k$ such that $I \subset I_{k}$ properly. Then $I \subset I_{k}^{-}$or $I \subset I_{k}^{+}$and $\alpha-w$ is constant on $I$.

(ii) There exists a $k$ such that $I=I_{k}$. Then $\alpha-w$ is constant on both $I_{k}^{-}, I_{k}^{+}$.

(iii) There exists a $k$ such that $I_{k} \subset I$ properly. We denote by $k_{0}$ minimal such $k$. Let $J^{\#}$ denote the immediate dyadic parent of $J$. Then $I_{k_{0}}^{\#}=\left[\frac{2^{k_{0}}-2}{2^{k_{0}}}, 1\right] \subset I$. If $I_{k_{0}}^{\#} \subset I$ properly, than $I_{k_{0}-1} \subset\left(I_{k_{0}}^{\#}\right)^{\#} \subset I$, contradicting the definition of $k_{0}$. Hence $I=I_{k_{0}}^{\#}, I_{k_{0}} \subset I, 2\left|I_{k_{0}}\right|=|I|$ and $\alpha-w$ takes exactly two values on $I$. We note that both values are taken on $I_{k_{0}}^{-}, I_{k_{0}}^{+}$.

To summarize, $I$ either is contained within some $I_{k}$, or contains a number of $I_{k}$ 's in their entirety. In either case the function $\alpha-w$ on $I$ is constant, or assumes exactly 2 values, spread over sets of equal measure. In any case, the norm equivalence condition (6) is immediate.

The following two theorems have motivated the construction of the counterexample in Theorem 1. We recall that we work with the weight $w$ constructed for a fixed $n$.

Theorem 2 If $\mathcal{M} f \in L^{1}([0,1])$, supp $f \subset[0,1], \int f=\int w f=0$, then $f$ admits decomposition

$$
f=\sum_{I-\text { dyad. }} \lambda_{I} a_{I}
$$

where, for some constant $C$ independent on $n$

$$
\sum_{I}\left|\lambda_{I}\right| \leq C\|\mathcal{M} f\|_{L^{1}}
$$

and $a_{I}$ are atoms, satisfying double cancellation condition

$$
\int a_{I}=\int a_{I} w=0
$$

Proof We note that the argument we use in the proof is standard, the only difference is in the cancellation statements involved. We begin with a definition of an auxiliary maximal operator $\mathcal{M}^{*}$, playing the role of the classical grand maximal operator.

$$
\mathcal{M}^{*} f(x)=\sup _{\substack{I \subset[0,1], I \text { dyad. } \\ \alpha \in \mathbb{R}}} \frac{\mathbb{1}_{10 I}(x)}{|I| \cdot\|\alpha-w\|_{L^{\infty}(I)}}\left|\int_{I} f \cdot(\alpha-w)\right| .
$$

It is an easy consequence of the definition of $\mathcal{M}$ and $\mathcal{M}^{*}$ that

$$
\left|\left\{x: \mathcal{M}^{*} f(x)>\lambda\right\}\right| \leq 10|\{x: \mathcal{M} f(x)>\lambda\}| .
$$

If $x \in\left\{x: \mathcal{M}^{*} f(x)>\lambda\right\}$ than $x \in 10 I$ for some dyadic interval $I$ such that $I \subset\{x: \mathcal{M} f(x)>\lambda\}$. Now, if we write

$$
\{x: \mathcal{M} f(x)>\lambda\}=\bigcup \tilde{I}
$$


where $\tilde{I}$ are maximal dyadic. Thus each $I \subset \tilde{I}$ for some $\tilde{I}$ and consequently

$$
\left|\left\{x: \mathcal{M}^{*} f(x)>\lambda\right\}\right| \leq 10|\{x: \mathcal{M} f(x)>\lambda\}| .
$$

The immediate corollary is

$$
\left\|\mathcal{M}^{*} f\right\|_{L^{1}} \leq C\|\mathcal{M} f\|_{L^{1}}
$$

For the dyadic interval $I$ we denote by $P_{I}(f)$ the orthonormal projection of $f$ onto the space spanned by the weights $\mathbb{1}_{I}$ and $\mathbb{1}_{I} \cdot w$

$$
P_{I}(f)(x)=\frac{\mathbb{1}_{I}(x)}{|I|} \int_{I} f(y) d y+\frac{\Delta_{I}(x)}{\left\|\Delta_{I}\right\|_{L^{2}}^{2}} \int_{I} f(y) \Delta_{I}(y) d y .
$$

We observe that

$$
\left\langle P_{I}(f), 1\right\rangle=\left\langle f \cdot \mathbb{1}_{I}, 1\right\rangle, \quad \text { and }\left\langle P_{I}(f), w\right\rangle=\left\langle f \mathbb{1}_{I}, w\right\rangle,
$$

directly by the definition (7). Denote by $A_{s}$ the set $\left\{\mathcal{M}^{*} f(x)>2^{s}\right\}$ and let $A_{1}=\bigcup_{r_{1}} I_{r_{1}}$ be the Whitney decomposition. Since by the construction $A_{s+1} \subset A_{s}$ so we can choose the Whitney decomposition $A_{2}=\bigcup_{r_{1}, r_{2}} I_{r_{1}, r_{2}}$ in such a way that $I_{r_{1}, r_{2}} \subset I_{r_{1}}$. We continue this process, obtaining of a tree of dyadic intervals $\left\{I_{r_{1}}, \cdots, I_{r_{1}, r_{2}, \cdots, r_{s}}, \cdots\right\}$. We write

$$
\begin{aligned}
f(x)= & \sum_{s} \sum_{r_{1}, r_{2}, \cdots, r_{s}}\left(\left(\mathbb{1}_{I_{r_{1}, r_{2}, \cdots, r_{s}}}(x)-\sum_{r_{s+1}} \mathbb{1}_{I_{r_{1}, r_{2}}, \cdots, r_{s}, r_{s+1}}(x)\right) \cdot f(x)-\right. \\
& \left.-P_{I_{r_{1}, r_{2}, \cdots, r_{s}}}(f)(x)+\sum_{r_{s+1}} P_{I_{r_{1}, r_{2}, \cdots, r_{s}, r_{s+1}}}(f)(x)\right)
\end{aligned}
$$

Each component

$$
\begin{aligned}
b_{I_{r_{1}, r_{2}, \cdots, r_{s}}}(x)= & \left(\mathbb{1}_{I_{r_{1}, r_{2}, \cdots, r_{s}}}(x)-\sum_{r_{s+1}} \mathbb{1}_{\left.I_{r_{1}, r_{2}, \cdots, r_{s}, r_{s+1}}(x)\right) \cdot f(x)-}\right. \\
& -P_{I_{r_{1}, r_{2}, \cdots, r_{s}}}(f)(x)+\sum_{r_{s+1}} P_{I_{r_{1}, r_{2}, \cdots, r_{s}, r_{s+1}}}(f)(x)
\end{aligned}
$$

satisfies

$$
\begin{aligned}
& \left|b_{I_{r_{1}, r_{2}, \cdots, r_{s}}}(x)\right| \leq C 2^{s} \\
& \sum_{s} \sum_{r_{1}, r_{2}, \cdots, r_{s}} 2^{s}\left|I_{r_{1}, r_{2}, \cdots, r_{s}}\right| \leq C\left\|\mathcal{M}^{*} f\right\|_{L^{1}} \\
& \left\langle b_{I_{r_{1}, r_{2}, \cdots, r_{s}}}, \mathbb{1}_{I}\right\rangle=0, \quad\left\langle b_{I_{r_{1}, r_{2}, \cdots, r_{s}}}, \Delta_{I}\right\rangle=0 .
\end{aligned}
$$


This means that

$$
a_{I_{r_{1}, r_{2}, \cdots, r_{s}}}(x)=\frac{b_{I_{r_{1}, r_{2}, \cdots, r_{s}}}(x)}{2^{s}\left|I_{r_{1}, r_{2}, \cdots, r_{s}}\right|}
$$

are double cancellation atoms and

$$
f(x)=\sum \lambda \lambda_{I_{r_{1}, r_{2}, \cdots, r_{s}}} a_{I_{r_{1}, r_{2}, \cdots, r_{s}}}(x),
$$

where

$$
\sum\left|\lambda_{I_{r_{1}, r_{2}, \cdots, r_{s}}}\right| \leq C\left\|\mathcal{M}^{*} f\right\|_{L^{1}}
$$

The theorem follows.

We leave it to the reader to extend Theorem 2 to any pair $w_{1}, w_{2}$ having the property that $w=w_{2} w_{1}^{-1}$ satisfies (6).

Corollary Assume that $w_{1}, w_{2}$ are polynomials satisfying $1 \leq w_{1}, w_{2} \leq C$ on $[0,1]$ and that $f \in \mathbb{H}^{1}$, supp $f \subset[0,1]$ is simultaneously orthogonal to $w_{1}$ and $w_{2}$. Then $f$ admits an atomic decomposition with atoms simultaneously orthogonal to $w_{1}, w_{2}$.

Proof The proof is based on standard norm-comparison properties for polynomials . We need to check the assumption of the Theorem 2 for polynomials $w_{1}$ and $w_{2}$.

If $J \subset[0,1]$ is an interval, $|J|=R$ and $w$ is a polynomial of degree $d$ (here $\left.w=\alpha w_{1}-w_{2}\right)$, then

$$
\sup _{x \in J}|w(x)| \approx \sum_{k=0}^{d} R^{k}\left|w^{(k)}(a)\right|
$$

The implied constants in (9) do not depend on $R$ and $a \in J$. Hence, for any $I \subset J$, we have $\sup _{x \in J}|w(x)| \geq C R \sup _{x \in I}\left|w^{(1)}(x)\right|$ again with the constant $C$ independent of $I$ and $J$. With this observation the estimate for $\mathcal{M} a, a$ being a classical $\mathbb{H}^{1}$ atom, is an application of the standard cancellation argument. We note, that the proof of the corollary can be made independent of Theorem 2. We leave details to the reader.

We present one more result.

Theorem 3 Suppose $f \in w_{1} \mathbb{H}^{1} \cap w_{2} \mathbb{H}^{1}$. Then there exist coefficients $\left\{\lambda_{r_{1}, r_{2}, \ldots, r_{s}}: r_{i} \in\right.$ $J_{i}-$ finite, $\left.s \in \mathbb{N}\right\}$ and a tree of dyadic intervals $\left\{I_{r_{1}, r_{2}, \ldots, r_{s}}: r_{i} \in J_{i}-\right.$ finite, $\left.s \in \mathbb{N}\right\}$, with inclusions

$$
I_{r_{1}} \supset I_{r_{1}, r_{2}} \supset \cdots \supset I_{r_{1}, r_{2}, \ldots, r_{s}} \supset \ldots
$$

such that $f$ admits atomic decompositions

$$
f=\sum_{\substack{r_{i} \in I_{i}, i=1, \ldots s \\ s \in \mathbb{N}}} \lambda_{r_{1}, r_{2}, \ldots, r_{s}} b_{r_{1}, r_{2}, \ldots, r_{s}}^{j}, \quad j=1,2,
$$


where the atoms are given by

and

$$
\begin{aligned}
b_{r_{1}, r_{2}, \ldots, r_{s}}^{j}(x)= & \frac{1}{\lambda_{r_{1}, r_{2}, \ldots, r_{s}}}\left(f \cdot \mathbb{1}_{I_{r_{1}, \ldots, r_{s}} \backslash \bigcup_{r_{s+1}} I_{r_{1}, \ldots, r_{s+1}}}(x)\right. \\
& \left.-\langle f\rangle_{I_{r_{1}, \ldots, r_{s}}, w_{j}}+\sum_{r_{s+1}} \mathbb{1}_{I_{r_{1}, \ldots, r_{s+1}}}(x)\langle f\rangle_{I_{r_{1}, \ldots, r_{s+1}}, w_{j}}\right),
\end{aligned}
$$

$$
\langle f\rangle_{I, w}=\frac{1}{|I|} \int_{I} f \cdot w .
$$

We omit the proof which is similar to the classical case of dyadic atomic decomposition. The argument yielding Theorem 2 can be adapted here as well. The only change required is to consider level sets of $\mathcal{M}_{1}^{*}\left(w_{1} \cdot f\right)+\mathcal{M}_{1}^{*}\left(w_{2} \cdot f\right)$, for $\mathcal{M}_{1}^{*}$ being the dyadic grand maximal operator

$$
\mathcal{M}^{*} f(x)=\sup _{\substack{I \subset[0,1], I-\text { dyad. } \\ \alpha \in \mathbb{R}}} \frac{\mathbb{1}_{10 I}(x)}{|I|}\left|\int_{I} f\right| .
$$

We leave details to the reader.

Open Access This article is licensed under a Creative Commons Attribution 4.0 International License, which permits use, sharing, adaptation, distribution and reproduction in any medium or format, as long as you give appropriate credit to the original author(s) and the source, provide a link to the Creative Commons licence, and indicate if changes were made. The images or other third party material in this article are included in the article's Creative Commons licence, unless indicated otherwise in a credit line to the material. If material is not included in the article's Creative Commons licence and your intended use is not permitted by statutory regulation or exceeds the permitted use, you will need to obtain permission directly from the copyright holder. To view a copy of this licence, visit http://creativecommons.org/licenses/by/4.0/.

\section{References}

1. Bernard, A., Maisonneuve, B.: Décomposition atomique de martingales de la classe $H^{1}$, Séminaire de Probabilités XI (Lect. Notes Math., vol. 581, pp. 303-323) Berlin, Heidelberg, New York. Springer (1977)

2. Coifman, R.R.: A real variable characterization of $H^{p}$. Studia Math. 51, 269-274 (1974)

3. Herz, C.S.: $H^{p}$ spaces of martingales, $0<p \leq 1$. Zeit f. War. 28, 189-205 (1974)

4. Herz, C.S.: Bounded mean oscillation and regulated martingales. Trans. Am. Math. Soc. 193, 199-215 (1974)

5. Latter, R.H.: A decomposition of $H^{p}\left(\mathbb{R}^{n}\right)$ in terms of atoms. Studia Math. 62, 92-101 (1978)

6. Stein, E.M.: Harmonic Analysis: Real-Variable Methods, Orthogonality and Oscillatory Integrals. Princeton University Press, Princeton (1993)

7. Stein, E.M., Weiss, G.L.: Introduction to Fourier Analysis on Euclidean Speces. Princeton University Press, Princeton (1971)

8. Weisz, F.: Martingale Hardy Spaces and their Applications in Fourier Analysis, (Lect. Notes Math., vol. 1568) Berlin, Heidelberg, New York. Springer (1994)

Publisher's Note Springer Nature remains neutral with regard to jurisdictional claims in published maps and institutional affiliations. 